\title{
SIMPLE GEODESICS ON RIEMANN SURFACES
}

\author{
TROELS JORGENSEN ${ }^{1}$
}

\begin{abstract}
As observed by Myrberg, on any closed hyperbolic surface the closed geodesics cover a dense subset. Below, it is shown that the simple geodesics cannot cover such parts of a surface which are sufficiently thin.
\end{abstract}

1. Introduction. Even though surfaces are rather well understood there remain seemingly difficult problems to study, many of which are related to the works of Nielsen and Thurston. One question, asked by W. Abikoff, is if the simple closed geodesics on a closed hyperbolic surface fill up a dense subset of the surface. The answer is generally in the negative.

THEOREM. For any hyperbolic surface of finite area, there exists a hyperbolic surface of the same signature, which is not covered by its simple geodesics.

The proof is elementary. It involves the concept of geometric convergence of Fuchsian groups combined with an observation about simple geodesics near punctures.

It is plausible that no hyperbolic surface is covered by its simple geodesics. It might even be that the set which is covered always has measure zero. This problem does not seem touchable by elementary methods nor by techniques from ergodic theory. Perhaps a careful analysis of Thurston's "train tracks" [8] will lead to the solution.

2. Geometric convergence. A hyperbolic surface may be thought of as the quotient surface $\mathbf{H} / G$ of the hyperbolic plane $\mathbf{H}$ under a Fuchsian group $G$. An introduction to the theory of Fuchsian groups may be found in $[2,3,4$ or 6].

A sequence of Fuchsian groups $\left\{G_{n}\right\}$ converges geometrically to the group $G$ if and only if

(i) each element $g \in G$ is the limit of a sequence of elements $g_{n} \in G_{n}$ (converging uniformly on compact subsets of $\mathbf{H}$ ) and

(ii) for any subsequence $\left\{F_{n}\right\}$ of $\left\{G_{n}\right\}$ and any converging sequence of elements $f_{n} \in F_{n}$, the limit $f=\lim _{n \rightarrow \infty} f_{n}$ belongs to $G$.

This is a useful notion of convergence. It goes back to Chabauty [1] and has been studied by Harvey [5] and Mumford [7] among others.

If $G$ is a Fuchsian group and $l$ is a hyperbolic line then one says that $l$ is simple modulo $G$ if and only if the intersection of $l$ and $g(l)$ is not a point for any $g \in G$. This amounts to the same as saying that the projection of $l$ on $\mathbf{H} / G$ is a simple geodesic, that is, a geodesic without proper self-intersections.

The set of all lines containing a given point in $\mathbf{H}$ is compact, and thus, the following is obvious.

Received by the editors July 23, 1981.

1980 Mathematics Subject Classification. Primary 53A35.

${ }^{1}$ Supported in part by the National Science Foundation and by the Alfred P. Sloan Foundation. 0002-9939/81/0000-0182/801.75 
LEMMA 1. Let $\left\{l_{n}\right\}$ be a sequence of hyperbolic lines converging to the line $l$, and let $\left\{G_{n}\right\}$ be a sequence of Fuchsian groups converging geometrically to the group $G$. If $l_{n}$ is simple modulo $G_{n}$, then $l$ is simple modulo $G$.

Applying this with all the $G_{n}$ 's equal, one obtains

COROLLARY. On any hyperbolic surface, the points belonging to simple geodesics form a closed set.

3. Punctures and branch points. Fuchsian groups may contain elliptic and parabolic elements. Such appear when the quotient surfaces have branch points or punctures.

LEMMA 2. Let $S$ be a hyperbolic surface and let $p \in S$ be a puncture or a branch point of order at least 3 . There exists a neighborhood of $p$ which contains a nonempty open subset of points not belonging to any simple geodesic on $S$.

The proof actually yields a little more information. Namely, the neighborhood can be chosen such that each simple geodesic entering it passes through the point $p$.

Suppose that $p$ is a puncture. In this case it is convenient to represent $S$ as the quotient surface of a Fuchsian group $G$, acting on the upper half-plane. Specifically, $S$ may be thought of as a fundamental polygon $P$ for $G$. We may also assume that $\infty$ is a lift of $p$ and that $P$ near $\infty$ is a vertical strip. The other sides of $P$ can be taken as arcs of isometric circles of certain elements in $G$ (see [3]).

In $G$, the stabilizer of $\infty$ is a cyclic, parabolic group. By conjugation it can be achieved that it is generated by the translation by 1 . When this is so, any hyperbolic line of euclidian radius more than $\frac{1}{2}$ meets its translate and thus cannot be simple modulo $G$. Hence, the half-plane of points abouve height $\frac{1}{2}$ only meets those simple geodesics that pass through $\infty$. This half-plane projects onto a neighborhood of $p$ on $S$.

Let $e$ be a nonvertical side of $P$ of maximal euclidian radius, and let $e^{\prime}$ be the side of $P$ equivalent to $e$. Together, the two half-lines $l$ and $l^{\prime}$ connecting $\infty$ and the respective top points of $e$ and $e^{\prime}$ project onto a simple geodesic on $S$. However, in a sufficiently narrow strip about $l$, any vertical line from $\infty$ to $e$ has as its geodesic continuation an arc of radius more than $\frac{1}{2}$ issuing from $e^{\prime}$. Thus, no simple geodesics pass through the points above height $\frac{1}{2}$ in this narrow strip, except $l$ itself. This, together with the corollary to Lemma 1 , implies Lemma 2, when $p$ is a puncture.

If $p$ is an elliptic branch point of order at least 3 , then an analogous argument applies, using as a model of $\mathbf{H}$ the unit disk instead of the upper half-plane, and placing a lift of $p$ at the origin. This completes the proof.

4. Moduli. For most hyperbolic surfaces the metric is not unique. The different structures on a given surface form the space of moduli. Uniqueness occurs only for spheres with 3 distinguished points, punctures or branch points. If all 3 points are branch points, then at least one of them has order more than 3 . Therefore, for all these rigid surfaces the theorem follows from Lemma 2.

Once the moduli space contains more than one point, it is not even compact. This was already known to Friche and Klein [4]. The lack of compactness, in case of surfaces of finite area, stems from the possibility of making simple closed geodesics 
arbitrarily short, as shown by Mumford [7]. Yet, some compactness does appear for surfaces of finite area.

Consider a sequence $\left\{S_{n}\right\}$ of topologically identical surfaces with different finite hyperbolic metrics. It was shown in [5] that there exists a sequence $\left\{G_{n}\right\}$ of Fuchsian groups such that $\mathbf{H} / G_{n}$ is isometric to $S_{n}$ for each $n \in \mathbf{N}$, and such that a subsequence of $\left\{G_{n}\right\}$ converges geometrically to a Fuchsian group $G$. If $S_{n}$ is chosen such that the length of some loop decreases to zero as $n$ tends to infinity, then it can be assumed that $\mathbf{H} / G$ is a punctured surface. Hence, using Lemma 1 , we conclude that the set of sufaces topologically isomorphic to $S_{n}$, which are covered by their simple geodesics, must lie within a geometrically compact subset of the moduli space. In particular, this proves the theorem. With a little more effort, the conclusion of the theorem can be verified for arbitrary hyperbolic surfaces.

\section{REFERENCES}

1. C. Chabauty Limite d'ensembles et géométrie des nombres, Bull. Soc. Math. France 78 (1950), 143-151.

2. W. Fenchel and J. Nielsen, Treatise on Fuchsian groups, manuscript.

3. L. R. Ford, Automorphic functions, 2nd ed., Chelsea, New York, 1951.

4. R. Fricke and F. Klein, Vorlesungen über die Theorie der Automorphen Funktionen, Leipzig, 1926.

5. W. F. Harvey, Chabauty spaces of discrete groups, Ann. of Math. Studies, no. 79, Princeton Univ. Press, Princeton, N. J., 1974, pp. 239-249.

6. J. Lehner, Discontinuous groups and automorphic functions, Math. Surveys, Vol. 8, Amer. Math. Soc., Providence, R. I., 1964.

7. D. Mumford, A remark on Mahler's compactness theorem, Proc. Amer. Math. Soc. 28 (1971), 289-294.

8. W. Thurston, The geometry and topology of 3-manifolds, Lecture notes, Princeton Univ., 197778.

DePARTMENT OF MATHEMATICS, UNIVERSity OF MinNesota, MinNeapolis, MiNNESOTA 55455

Current address: Department of Mathematics, Columbia University, New York, New York 10027 